\title{
It Is the Time to Think About a Treat-to-Target Strategy for Knee Osteoarthritis
}

This article was published in the following Dove Press journal:

Therapeutics and Clinical Risk Management

\author{
A Migliore (iD)' \\ G Gigliucci \\ RJ Petrella ${ }^{2}$ \\ RR Bannuru (iD) 3 \\ $X$ Chevalier (D) ${ }^{4}$ \\ E Maheu $^{5}$ \\ R Raman ${ }^{6}$ \\ G Herrero-Beaumont ${ }^{7}{ }^{7}$ \\ $\mathrm{N}$ Isailovic ${ }^{8}$ \\ M Matucci Cerinc ${ }^{9}$
}

'Rheumatology Unit, San Pietro Fatebenefratelli Hospital, Rome, Italy;

${ }^{2}$ Department of Family Medicine, School of Kinesiology Western University, Western Centre for Public Health \& Family, London, Ontario, Canada; ${ }^{3}$ Center for Treatment Comparison and Integrative Analysis Division of Rheumatology, Tufts Medical Center, Boston, MA, USA; ${ }^{4}$ Department of Rheumatology, Hôpital Henri Mondor, Creteil, France; ${ }^{5}$ Rheumatology Department, AP-HP, Saint-Antoine Hospital, Paris, France; ${ }^{6}$ Academic Department of Orthopaedics, Hull and East Yorkshire NHS Trust, Castle Hill Hospital, Cottingham, UK; ${ }^{7}$ Joint and Bone Research Unit, IIS-Fundacion Jimenez Diaz UAM, Madrid, Spain; ${ }^{8}$ Division of Rheumatology and Clinical Immunology, Humanitas Research Hospital, Rozzano, Milan, Italy; ${ }^{9}$ Division of Rheumatology AOUC, Department of Experimental and Clinical Medicine, University of Florence, Florence, Italy

Correspondence: $\mathrm{N}$ Isailovic Division of Rheumatology and Clinical Immunology, Humanitas Research Hospital, Via A. Manzoni 56, Rozzano 20089, Milan, Italy

Tel +39-02-8224-5II8

Email natasa.isailovic@humanitasresearch.it
Abstract: Osteoarthritis (OA) is a rheumatic disease that affects the well-being of the patient, compromises physical and mental function, and affects other quality of life aspects. In the literature, several evidence-based guidelines and recommendations for the management of knee osteoarthritis (KOA) are available. These recommendations list the different therapeutic options rather than addressing a hierarchy between the treatments and defining the real target. Therefore, a question arises: are patients and physicians satisfied with the current management of KOA? Actually, the answer may be negative, thus suggesting a change in our therapeutic strategies. In this article, we address this challenge by suggesting that it is time to develop a "treat to target strategy" for KOA.

Keywords: osteoarthritis, knee osteoarthritis, treat to target

\section{Introduction}

Osteoarthritis (OA) is the most common rheumatic disease, with an estimated overall prevalence, in the general adult population, of $11 \%$ and $24 \%$ for hip and knee OA, respectively. ${ }^{1}$ Defined on the basis of symptoms and physical findings, approximately 27 million US adults and 8.5 million UK adults are estimated to be affected by symptomatic OA. ${ }^{2}$ In elderly people, OA is the most common cause of disability, including pain and limitations of activities and social participation. ${ }^{3-5}$ Soon, the number of people living with severe longstanding OA will significantly grow as a result of the increase in life expectancy and increasing risk factors of OA such as obesity and sedentary lifestyle.

What have we learned from the management of other chronic diseases? Clearly, we have learned the importance and relevance of finding a therapeutic target considering disease activity, severity and meeting patient expectation.

For many chronic diseases, there are specific targets and established surrogate markers useful for monitoring disease evolution over time and driving the treatment. For example, in diabetic patients, it is crucial to keep within specific limits of blood glucose level or glycated hemoglobin serum level to better control the disease and to prevent diabetes-related complications. In osteoporotic women, the evaluation of bone mass index (bone mineral density (BMD)) and prevalent or incident vertebral fractures drive the treatment target to prevent new osteoporotic fractures. In patients at high risk of cardiovascular events, the targets are to maintain normal blood pressure values to reach normal serum lipid levels (total cholesterol, LDL cholesterol, HDL cholesterol, triglycerides) and to normalize weight to prevent cardiovascular or ischemic complications. Although many therapies are now 
available for OA, there is still an enormous need for diagnosis and target biomarkers that can lead to more concrete treat-to-target strategy. Until then, our focus can be directed to treat-to-target strategy for KOA similar to the one we have for management of rheumatoid arthritis (RA).

In RA, the treat-to-target strategy, based on the definition of a specific target (remission or low disease activity calculated by the level of disease Activity Score 28 (DAS28)), has been developed along with a detailed therapeutic strategy aiming to achieve this target through a tight disease control. The DAS28 counts the number of painful and swollen joints, biological inflammation indexes and patient overall status, providing, also in everyday practice, a measure disease activity. ${ }^{6}$

Recently, the treat-to-target strategy has significantly ameliorated the management of patients affected by RA and improved adherence to therapies and the prevention of structural damage.

Current treatment options in OA remain limited and controversial. Conventional RA DMARDs have not been demonstrated beneficial in managing OA symptoms. However, recent clinical trials involving peripheral nociceptive targets, such as Tanezumab, monoclonal antibody against nerve growth factor has a promising analgesic effect in KOA. Furthermore, the two studies of both a cartilage anabolic agent (Sprifermin (rhFGF18)) and an osteoclast inhibitor suggest a potential for structural bone modification in OA affected patients but without symptom benefits so far. This lack of achieving primary endpoints may be due to a short duration of follow-up in these OA clinical trials. A number of these putative diseasemodifying osteoarthritis drugs (DMOADs) are in phase II development, but preliminary results demonstrate no concomitant symptom improvement, raising new considerations for future DMOAD trials. ${ }^{7}$

These therapeutic strategies are based on shared choices between patient and clinician and are achieved with a regular monitoring of disease activity, considering comorbidities and other relevant clinical factors. ${ }^{6}$

In KOA, the introduction of a shared strategy based on a treat-to-target principle might become in the next future the goal to achieve an optimal management. The main goals in OA need to be defined. A treat-to-target strategy should aim at improving joint pain and quality of life especially in elderly population. This approach may help to find the best combination/sequence of the most appropriate treatments to reach this target for any patient.
This approach could help us to surpass the limits of the international recommendations. Despite several guidelines for KOA treatment are available, ${ }^{8,9}$ many unmet needs remain unresolved. Most importantly, many items still await to be clarified like the identification of a clear target of shared parameters to define the effectiveness of a treatment; the lack of suggestions on how to manage the disease on the long term and managing the flare of the disease as well as the absence of a personalized therapy according to patient characteristics and/or OA phenotypes.

Actually, the international recommendations for KOA management summarize treatments into three categories: non-pharmacological, pharmacological and surgical. In particular, the European League Against Rheumatism (EULAR) produced evidence-based recommendations for the non-pharmacological management of hip or knee OA. ${ }^{8}$ These recommendations focused on a patient-centered multidisciplinary approach to the management including patient education to achieve an appropriate lifestyle. Also the OA Research Society International (OARSI) published an update of evidence-based, consensus recommendations for the KOA treatment. ${ }^{9}$ These recommendations were intended to optimize in practice KOA therapy based on expert review of safety and efficacy of the available treatments. The aim of OARSI was to develop a precise algorithm considering the involvement of single or multiple joints and the presence or absence of comorbidity to guide the clinician in choosing the most correct therapeutic approach.

In 2014, a task force of the European Society for Clinical and Economic Aspects of Osteoporosis and Osteoarthritis (ESCEO) proposed an algorithm for KOA management. Tthe 2014 version of the NICE (National Institute for Health and Care Excellence) guideline for care and management in osteoarthritis patients offered implementation tools and resources to help users put the recommendations into practice. ${ }^{10}$ Combined pharmacological and non-pharmacological options were considered with a core set of initial treatments, including information access/education, weight loss and an appropriate exercise program. Four multimodal steps were established; based on non-pharmacological treatment (i.e., physical interventions) or sustained symptomatic slow-acting drugs for OA (e.g., glucosamine sulfate and/or chondroitin sulfate), paracetamol, topical NSAIDs and advanced pharmacological management in the persistent symptomatic patient. ${ }^{11}$ This evidence-based algorithm provided a detailed sequence of pharmacological and non-pharmacological 
treatments considering the persistence of symptomatic OA and contraindications. These recommendations represent a starting point to improve the management of patients with KOA, but still they do not consider a treat-to-target approach.

Furthermore, the majority of randomized controlled trials that were used to support evidence-based guidelines include a follow-up of only 3 to 6 months which is a clear limitation regarding the management of a long-term disease such as OA. Mostly subjective parameters such as pain, function assessment scales like WOMAC or LEQUESNE indexes are used in the short term to evaluate the symptomatic clinical response. Few studies consider outcomes in the longer term. In fact, longer follow-up seems more appropriate to assess the therapeutic effect on several domains such as pain, function, quality of life and disease progression and total knee replacement (TKR) as a final hard outcome.

However, even if guidelines and recommendations are useful tools for clinicians, it now may be the right time to move a step forward into a treat-to-target strategy.

In this endeavor, a biomarker might help to target disease. Unfortunately, in $\mathrm{KOA}$, no specific biomarker is still available, significantly reducing our capacity to monitor the disease. At present, the characterization and qualification of various biomarkers are still ongoing. ${ }^{12}$ In the literature, several articles have focused on candidate pathways and molecules that seem promising for further understanding of OA pathophysiology and development of therapeutics. However, up to now, none is currently available in daily practice.

The most important unanswered question is: what is the ideal therapeutic target in OA? It is not yet clearly defined and standardized. The search for a clinical target remains an unmet need in KOA.

In clinical trials, usual endpoints are pain, function, range of motion, quality of life, structural evolution progression and even hard outcomes such as delay to TKR. In addition, in KOA, the tissues of the whole joint (bone, muscle, cartilage, synovia and joint capsule) are involved to varying extents according to the phenotype and progression of the disease. Therefore, it is difficult to identify or to suggest a single target from a structural prospective. The OA management should consider the long duration and the different phases of the disease activity together with several specific phenotypes during its course. ${ }^{13,14}$

Moreover, it is very difficult to evaluate the delay to TKR because of the lack of standardized indication, biomarkers, surrogate parameters and trials with a long follow-up. Regarding imaging, only radiographic parameters are currently well standardized to evaluate the structural progression. Magnetic resonance imaging, despite interesting results in terms of predictive parameters of disease progression, does not provide well-standardized assessment tools or parameters.

In addition, a long follow-up period is essential for the evaluation of both clinical and structural parameters.

Besides the target to achieve, it would also be important to evaluate the effect-size to reach. For example, clinically, the level of pain or functional impairment acceptable by the patient (Patient Acceptable Symptom State-PASS) ${ }^{15}$ and regarding structural progression, stopping or slowing down radiographic progression, using a clinically significant threshold. ${ }^{16}$

In this context, an early KOA diagnosis might significantly impact the disease evolution, preventing the loss of quality of life and joint function. Recently an international panel of specialists proposed a definition of Early Symptomatic Knee OA (ESKOA) with the aim to identify patients affected by symptomatic KOA before the onset of radiological damage. ${ }^{17}$

In conclusion, lessons learned from the management of chronic diseases such as RA through a treat-to-target strategy, in the current context, represent an option that would help to respond to different unmet needs in OA. A number of existing pharmacological therapies have been recently trialed in OA with limited success in pain management and structural improvements.

In the future, a treat-to-target approach in the management of KOA might guide all practitioners involved in OA daily care, despite the difficulty to define the right targets in this multi-faceted disease, characterized by a long duration, chronic pain and long-term structural evolution, and in absence of a real efficient structure-modifying treatment.

Now, we believe that it is time to modify the strategy of KOA management, shifting the focus from available single therapeutic agents to a treat-to-target strategy.

For this purpose, a Technical Expert Panel promoted by the International Symposium on Intra Articular Treatment (ISIAT) has been commissioned to propose such a treat-to-target strategy for KOA.

\section{Acknowledgment}

The first author is grateful for enthusiastic support to all co-authors of this article. 


\section{Author Contributions}

All authors contributed to data analysis, drafting and revising the article, gave final approval of the version to be published, and agree to be accountable for all aspects of the work.

\section{Disclosure}

Professor Xavier Chevalier reports personal fees from Pfizer, Sanofi, and Ibsa, and hotel and travel expenses from Nordic Pharma, outside the submitted work. Dr Emmanuel Maheu reports personal fees from Expanscience, TRB Chemedica, MEDA - MYLAN, and Celgène, outside the submitted work. Dr Raghu Raman reports personal fees from AK medical and SANOFI, and personal fees and non-financial support from Macopharma, outside the submitted work. The authors report no other conflicts of interest in this work.

\section{References}

1. Pereira D, Peleteiro B, Araujo J, et al. The effect of osteoarthritis definition on prevalence and incidence estimates: a systematic review. Osteoarthritis Cartilage. 2011;19:1270-1285. doi:10.1016/j.joca.2011.08.009

2. Neogi T. The epidemiology and impact of pain in osteoarthritis. Osteoarthritis Cartilage. 2013;21(9):1145-1153. doi:10.1016/j.joca.20 13.03.018

3. Bijlsma JW, Berenbaum F, Lafeber FP. Osteoarthritis: an update with relevance for clinical practice. Lancet. 2011;377:2115-2126. doi:10.10 16/S0140-6736(11)60243-2

4. Felson DT, Lawrence RC, Dieppe PA, et al. Osteoarthritis: new insights. Part 1: the disease and its risk factors. Ann Intern Med. 2000;133:635-646. doi:10.7326/0003-4819-133-8-200010170-00016

5. Woolf AD, Pfleger B. Burden of major musculoskeletal conditions. Bull World Health Organ. 2003;81:646-656.

6. Smolen JS, Breedveld FC, Burmester GR, et al. Treating rheumatoid arthritis to target: 2014 update of the recommendations of an international task force. Ann Rheum Dis. 2016;75(1):3-15. doi:10.1136/ annrheumdis-2015-207524
7. Ghouri A, Conaghan PG. Update on novel pharmacological therapies for osteoarthritis. Ther Adv Musculoskelet Dis. 2019;11:1-11.

8. Fernandes L, Hagen KB, Bijlsma JW, et al. European League Against Rheumatism (EULAR) EULAR recommendations for the non-pharmacological core management of hip and knee osteoarthritis. Ann Rheum Dis. 2013;72(7):1125-1135. doi:10.1136/ annrheumdis-2012-202745

9. McAlindon TE, Bannuru RR, Sullivan MC, et al. OARSI guidelines for the non-surgical management of knee osteoarthritis. Osteoarthritis Cartilage. 2014;22(3):363-388. doi:10.1016/j.joca.2014.01.003

10. Meneses SR, Goode AP, Nelson AE, et al. Clinical algorithms to aid osteoarthritis guideline dissemination. Osteoarthritis Cartilage. 2016;24(9):1487-1499. doi:10.1016/j.joca.2016.04.004

11. Bruyère $\mathrm{O}$, Cooper $\mathrm{C}$, Pelletier JP, et al. An algorithm recommendation for the management of knee osteoarthritis in Europe and internationally: a report from a task force of the European society for clinical and economic aspects of osteoporosis and osteoarthritis (ESCEO). Semin Arthritis Rheum. 2014;44(3):253-263. doi:10.10 16/j.semarthrit.2014.05.014

12. Watt FE. Osteoarthritis biomarkers: year in review. Osteoarthritis Cartilage. 2018;26(3):312-318. doi:10.1016/j.joca.2017.10.016

13. Castaneda S, Roman-Blas JA, Largo R, Herrero-Beaumont G. Osteoarthritis: a progressive disease with changing pheno- types. Rheumatology. 2014;53(1):1e3. doi:10.1093/rheumatology/ket247

14. van der Esch M, Knoop J, van der Leeden M, et al. Clinical phenotypes in patients with knee osteoarthritis: a study in the Amsterdam osteoarthritis cohort. Osteoarthritis Cartilage. 2015;23(4):544-549. doi:10.1016/j.joca.2015.01.006

15. Bruyère $\mathrm{O}$, Cooper $\mathrm{C}$, Arden $\mathrm{N}$, et al. Can we identify patients with high risk of osteoarthritis progression who will respond to treatment? A focus on epidemiology and phenotype of osteoarthritis. Drugs Aging. 2015;32(3):179-187. doi:10.1007/s40266-015-0243-3

16. Tubach F, Ravaud P, Martin-Mola E, et al. Minimum clinically important improvement and patient acceptable symptom state in pain and function in rheumatoid arthritis, ankylosing spondylitis, chronic back pain, hand osteoarthritis, and hip and knee osteoarthritis: results from a prospective multinational study. Arthritis Care Res (Hoboken). 2012;64(11):1699-1707. doi:10.1002/acr.21747

17. Migliore A, Scirè CA, Carmona L, et al. The challenge of the definition of early symptomatic knee osteoarthritis: a proposal of criteria and red flags from an international initiative promoted by the italian society for rheumatology. Rheumatol Int. 2017;37 (8):1227-1236. doi:10.1007/s00296-017-3700-y
Therapeutics and Clinical Risk Management

\section{Publish your work in this journal}

Therapeutics and Clinical Risk Management is an international, peerreviewed journal of clinical therapeutics and risk management, focusing on concise rapid reporting of clinical studies in all therapeutic areas, outcomes, safety, and programs for the effective, safe, and sustained use of medicines. This journal is indexed on PubMed Central, CAS,
EMBase, Scopus and the Elsevier Bibliographic databases. The manuscript management system is completely online and includes a very quick and fair peer-review system, which is all easy to use. Visit http://www.dovepress.com/testimonials.php to read real quotes from published authors. 